\title{
Nanostructured ZnO coatings grown by pulsed laser deposition for optical gas sensing of butane
}

\author{
T. Mazingue ${ }^{a)}$ \\ Cybernétix, Technopôle de Château-Gombert, 306 rue Albert Einstein, BP 94-13382, \\ Marseille Cedex 13, France \\ L. Escoubas, L. Spalluto, and F. Flory \\ Institut Fresnel, Unité Mixte de Recherche (UMR) Centre National de la Recherche Scientifique (CNRS) \\ 6133, Ecote Généraliste d'Ingénieurs á Marseille (EGIM) Nord, Domaine Universitaire de Saint \\ Jérôme, 13397 Marseille Cedex 20, France
}

G. Socol, C. Ristoscu, E. Axente, S. Grigorescu, and I. N. Mihailescu ${ }^{\text {b) }}$

Lasers Department, National Institute for Lasers, Plasma and Radiations Physics, P.O. Box MG-54, RO-77125, Bucharest-Magurele, Romania

\section{N. A. Vainos \\ NHRF-The National Hellenic Research Foundation, Theoretical and Physical Chemistry Institute-TPCI, 48 Vasssileos Constantinou Avenue, Athens 11635, Greece and Department of Materials Science, University of Patras, Patras, 26504, Greece}

(Received 18 April 2005; accepted 29 August 2005; published online 12 October 2005)

\begin{abstract}
We report the detection of $100 \mathrm{ppm}$ of butane in superatmospheric $\mathrm{N}_{2}$ or air with an $m$-lines setup. The sensing elements are ZnO-nanostructured coatings prepared by pulsed laser deposition. The deposition technique was optimized to obtain highly transparent films of $1 \mathrm{~cm}^{2}$ in area and several hundreds nanometer thick. ZnO structures preserve gas sensitivity even when deposited at room temperature. Refractive index variations down to 0.005 were detected and typical variations of about $20 \%$ were induced during $\mathrm{ZnO}$ film-butane contact. (C) 2005 American Institute of Physics.
\end{abstract}

[DOI: 10.1063/1.2076442]

\section{INTRODUCTION}

Optical gas sensors have recently emerged as a very promising alternative to gas detectors based on electrical properties that currently dominate the market. The expected advantages of optical gas detectors over the classical electrical devices include simplicity, reliability, compactness, ruggedness, robustness, resistance to electromagnetic interference, and possibility to work at room temperature. A potential type of optical sensor makes use of thin planar waveguides. ${ }^{1-3}$

This paper reports experimental studies on the development of $\mathrm{ZnO}$ waveguides as optical gas sensing materials for the detection of hydrocarbons. $\mathrm{ZnO}$ is a remarkable material with a wide variety of optical, electrical, and piezoelectric applications. Because of its unique properties, $\mathrm{ZnO}$ is used in manufacturing solar cells, ${ }^{4}$ optical coatings, photocatalysts, electrical devices, and as an active medium in UV semiconductor lasers ${ }^{5}$ as well as in gas sensing. ${ }^{6-8}$ Several physical and chemical techniques such as molecular-beam epitaxy, ${ }^{9}$ chemical-vapor deposition, ${ }^{10}$ and sputtering ${ }^{11}$ have been applied for $\mathrm{ZnO}$ synthesis. Another versatile method, known as pulsed laser deposition (PLD), has also been widely used to produce nanostructured and textured $\mathrm{ZnO}$ films of high optical quality. ${ }^{12-15}$ It has been demonstrated that PLD provides some obvious advantages over the previous deposition methods through the control of deposition parameters, and conse-

\footnotetext{
${ }^{\text {a)} E l e c t r o n i c ~ m a i l: ~ t h o m a s . m a z i n g u e @ ~ f r e s n e l . f r ~}$

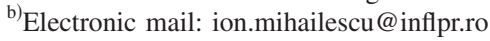

quently can significantly improve the overall characteristics of the films. Yet, neither the optical sensing capabilities of the obtained structures, nor their response to contact interactions with gases of technological interest have been explored so far.

We are reporting here the PLD growth of undoped $\mathrm{ZnO}$ coatings on $\mathrm{SiO}_{2}(001)$ substrates together with detection test results for different concentrations of butane in dry air and nitrogen by the $m$-lines technique.

\section{METHODS AND EXPERIMENTAL SETUPS}

\section{A. PLD synthesis}

$\mathrm{ZnO}$ thin films were coated on $\mathrm{SiO}_{2}(001)$ substrates by PLD (Fig. 1) using an excimer $\mathrm{KrF}^{*}$ laser source $(\lambda$

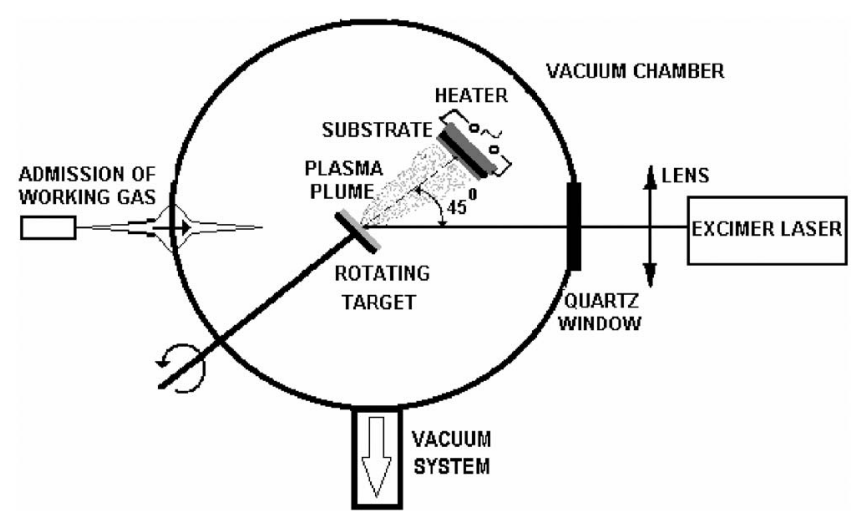

FIG. 1. PLD experimental setup. 

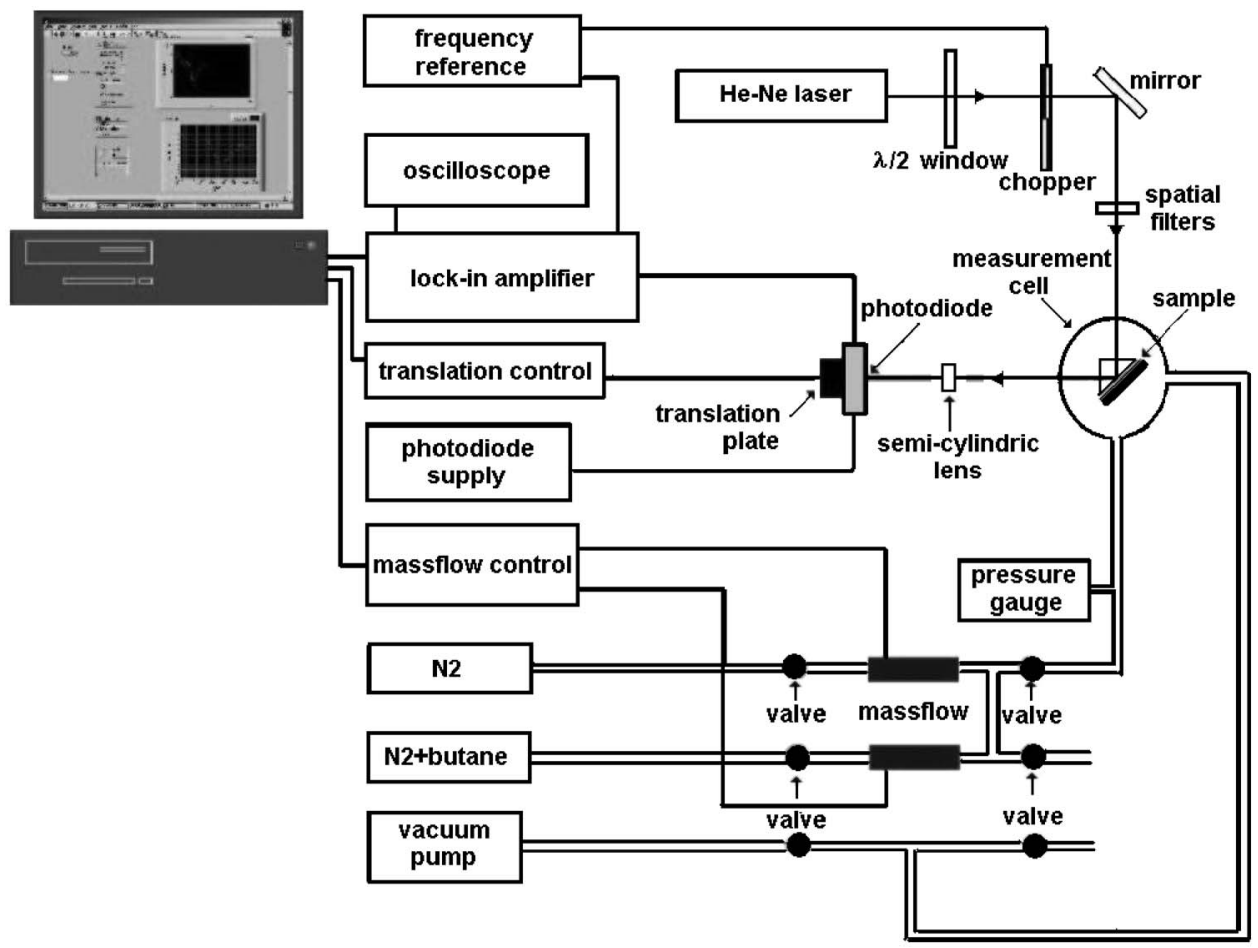

FIG. 2. Testing setup for waveguide coupling interrogation.

$=248 \mathrm{~nm}, \tau_{\mathrm{FWHM}} \approx 7 \mathrm{~ns}, \nu=2 \mathrm{~Hz}$ ), operated at a laser fluence of $2.6 \mathrm{~J} / \mathrm{cm}^{2}$ for a $5-\mathrm{cm}$ target-substrate distance. Prior to any deposition, the chamber was evacuated down to a residual pressure of $10^{-4} \mathrm{~Pa}$. The coatings were deposited from $\mathrm{ZnO}$ targets prepared from $\mathrm{ZnO}$ powder with $99.9 \%$ purity pressed at $20 \mathrm{MPa}$ and sintered at $1100{ }^{\circ} \mathrm{C}$ for $12 \mathrm{~h}$. The coatings were performed in a dynamic oxygen pressure assessed by an MKS 50 controller. To deposit one film, we applied 50000 subsequent laser pulses while the target was rotated with a frequency of $0.3 \mathrm{~Hz}$ to avoid piercing. During deposition, substrate temperature was kept at a constant value within the range of $20-350{ }^{\circ} \mathrm{C}$.

After deposition, the resulting structures were optically characterized with a double-beam spectrophotometer UVVIS (model Perkin Elmer Lambda 25) in the 300-1500 nm wavelength range. Structural and compositional investigations of these films performed with a DRON UM1 fully automated diffractometer in the $\theta-2 \theta$ mode were reported elsewhere. ${ }^{16}$ Our studies showed that the most transparent stoichiometric $\mathrm{ZnO}$ films were obtained in $\mathrm{O}_{2}$ pressure of $13 \mathrm{~Pa}$. The optimized deposition regime was further selected for the next experiments reported in this paper.

The main objective of this paper was to investigate the gas detection capabilities of the most transparent films. Special attention was paid to depositions performed at low temperatures, because of the self-regulation process governing grain size on the chemisorption of the gas onto $\mathrm{ZnO}$ coatings. ${ }^{17,18}$ Thus we tried to optimize the future compatibility of such films with other optical elements of interferometers, and develop a simple, reliable deposition technology with the lowest possible thermal budget.

\section{B. Determination of deposited films thickness and refractive index by the $m$-lines method}

The $m$-lines technique at $633 \mathrm{~nm}$ laser wavelength is used for refractive index and thickness measurement as well as an interrogation method for gas testing. This technique is suitable and frequently applied in order to measure the variations of optical parameters from a transparent waveguide. ${ }^{19-21}$ The experimental setup is presented in Fig. 2. At the center of the testing setup were the prism coupler on its rotation plate and the sample, i.e., the thin film, which was deposited on a $\mathrm{SiO}_{2}(001)$ substrate, pressed on the prism base. The physical interactions between the gas and the sensitive material leading to variations in the refractive index of the latter take place at the air-sensitive material interface. Calculations have shown that the electromagnetic field is enhanced at this interface for the TM polarization state of light and a thin-film thickness close to the cut-off thickness of the guided mode $^{22}$ (the thickness below which the mode cannot propagate in the thin film any longer). This configuration ensures the highest sensitivity to refractive index variation under gas exposure.

The coupling by a prism of an incident laser beam into a planar waveguide is governed by the incident angle $\theta_{s}$ of the beam on the prism base (Fig. 3). For a characteristic incident angle, the resonant coupling of the laser beam into the waveguide can be observed by detecting a dark line (known as mode line or $m$ lines) that appears in the reflected beam. Consequently, the method consists in measuring the angles

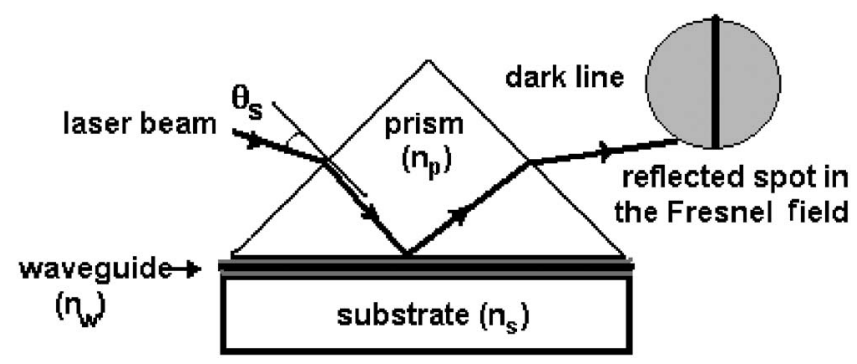

FIG. 3. Totally reflecting prism coupler. 
corresponding to the $m$ lines. From the propagation constants of the guided modes determined from these angles, we can obtain separately the refractive index $n$ and thickness $t$ of the waveguide. An angle measurement accuracy of $10^{-3}$, which is easily achieved, corresponds to an accurate $\left(10^{-3}\right)$ estimation of the refractive index $n$ and thickness $t$ of single layers $( \pm 2 \mathrm{~nm}){ }^{23}$

We studied the refractive indices and waveguiding properties of thin films for the two polarization states of light. The resonant coupling of the laser beam into the waveguide is strongly affected by a perturbation caused by an interaction of the material and surrounding atmosphere. The effective index $N$ of the guided mode corresponding to the resonant coupling is linked to the incident angle $\theta_{s}$ of light by Eq. (1),

$$
N=n_{p} \sin \left[\arcsin \left(\frac{\sin \theta_{s}}{n_{p}}\right)+A_{p}\right],
$$

where $n_{p}$ is the refractive index of the prism, and $A_{p}$ its characteristic angle.

From Eq. (1), we deduce the following expression of $\Delta N$ :

$$
\Delta N=\frac{n_{p} \cos \theta_{s} \cos \left[\arcsin \left(\sin \theta_{s} / n_{p}\right)+A_{p}\right]}{\sqrt{n_{p}^{2}-\left(\sin \theta_{s}\right)^{2}}} \Delta \theta_{s}
$$

\section{Optical gas detection}

The measuring arm was placed in front of the reflected beam at an angle corresponding to the $m$-lines position. A small reverse-voltage Hamamatsu ${ }^{\mathrm{TM}} \mathrm{Si}$ photodiode collecting the light was fixed to a motorized high-resolution $(1 \mu \mathrm{m})$ translation stage. The $m$-lines profile was recorded by a LABVIEW $^{\mathrm{TM}}$ computer application. If the refractive index of the material varied (for example, under gas exposure), the line was translated. The photodiode was therefore placed where the slope of the $m$-lines intensity curve was highest, in order to acquire the largest signal variation in case of optical sensitivity. The signal was amplified by a lock-in amplifier Stanford Research Systems SRS830, which was synchronized with a chopper to improve the signal-to-noise $(\mathrm{S} / \mathrm{N})$ ratio. We noted that the chopper frequency would have no influence on the optical response if $50 \mathrm{~Hz}$ harmonics were avoided. We therefore chose a frequency of $311 \mathrm{~Hz}$, which in addition was appropriate for observing phenomena in the temporal range of interest in our experiments (of the order of seconds).

The aim was to detect butane concentrations lower than $800 \mathrm{ppm}$ (or $1900 \mathrm{mg} / \mathrm{m}^{3}$ ), which is the maximum daily exposure authorized under U.S. Federal Regulations. ${ }^{24}$ As we found it is impossible to prepare a mixture with such precise concentrations. We chose, therefore, a ready-to-use mixture with $1000 \mathrm{ppm}$ of butane diluted in nitrogen $\left(\mathrm{N}_{2}\right)$. By mixing it with another bottle of $\mathrm{N}_{2}$, we managed to get concentrations $C$ from 100 to $1000 \mathrm{ppm}$, according to the following formula:

$$
C=\frac{D_{m}}{D_{m}+D_{\mathrm{N}_{2}}} C_{\mathrm{out} / \mathrm{N}_{2}},
$$

where $D_{m}$ is the flow of the mixture, $D_{\mathrm{N}_{2}}$ the flow of $\mathrm{N}_{2}$, and $\mathrm{C}_{\text {but } / \mathrm{N}_{2}}$ the concentration of butane diluted in nitrogen. $D_{m}$ and $D_{\mathrm{N}_{2}}$ were controlled by two numeric mass-flowmeters Aera FC7700CD. The desired concentration was sent into the cylindrical measurement cell made of transparent glass, which had been evacuated to remove impurities. The pressure was controlled by a Pirani gauge Inficon PSG400.

The valves prevented the gas mixture from returning to the setup upon vacuum break or a potential handling mistake (Fig. 2). The experimental protocol was as follows:

(i) evacuating the measurement cell to clear it from impurities (1 $\min 30)$,

(ii) filling the measurement cell with $\mathrm{N}_{2}$ (carrier gas) to atmospheric pressure (2 min 30),

(iii) introducing the mixture $\mathrm{N}_{2}+$ butane $(3 \mathrm{~min})$, and

(iv) stopping the mixture inflow and introducing $\mathrm{N}_{2}$ to study the return to base line $(3 \mathrm{~min})$.

We realized that gas cell dimensions would influence the response time of the sensor. Indeed, it will take longer to saturate the sensor-active sites inside a larger cell because the corresponding filling time is longer. We therefore made an optimized option for a 31 cell, but kept the gas flow constant at $11 \mathrm{~min}$ independently on the butane concentration, to get rid of the filling time influence.

\section{RESULTS AND DISCUSSION}

\section{A. Optical characterizations}

Our structural and morphological studies of $\mathrm{ZnO}$ films have evidenced characteristics quite dependent on substrate temperature. They further determined the optical parameters of the obtained films. Typical results for $\mathrm{ZnO}$ films obtained by PLD under optimal conditions are collected in Table I. They correspond to substrate temperatures of $350{ }^{\circ} \mathrm{C}$ (type A), $150{ }^{\circ} \mathrm{C}$ (type B), and RT (type C), respectively. All of the films, except the A-type samples for the TE polarization state, showed well-contrasted mode lines. The refractive indices of the $\mathrm{ZnO}$ films under investigation were in the region of 1.95-1.98. $m$-lines for the TE modes in the case of A-type films were too large and amply curved to allow an accurate calculation of $n_{\mathrm{TE}}$. The B- and C-type samples showed a slight anisotropy. A high substrate temperature during the deposition process led to a densification of the film resulting in a higher refractive index.

TABLE I. Optical properties of $\mathrm{ZnO}$ films deposited by PLD.

\begin{tabular}{ccccc}
\hline \hline $\begin{array}{c}\text { Sample } \\
\text { type }\end{array}$ & Modes & $n_{\mathrm{TE}}$ & $n_{\mathrm{TM}}$ & $\begin{array}{c}\text { Thickness } \\
(\mathrm{nm})\end{array}$ \\
\hline $\mathrm{A}$ & TE0, TE1, TE2, TE3, TM0, TM1, & $\mathrm{x}$ & 2.000 & 770 \\
& $\quad$ TM2, TM3 & & & \\
$\mathrm{B}$ & TE0, TE1, TE2, TM0, TM1, TM2 & 1.968 & 1.978 & 629 \\
$\mathrm{C}$ & TE0, TE1, TM0, TM1 & 1.955 & 1.952 & 435 \\
\hline \hline
\end{tabular}




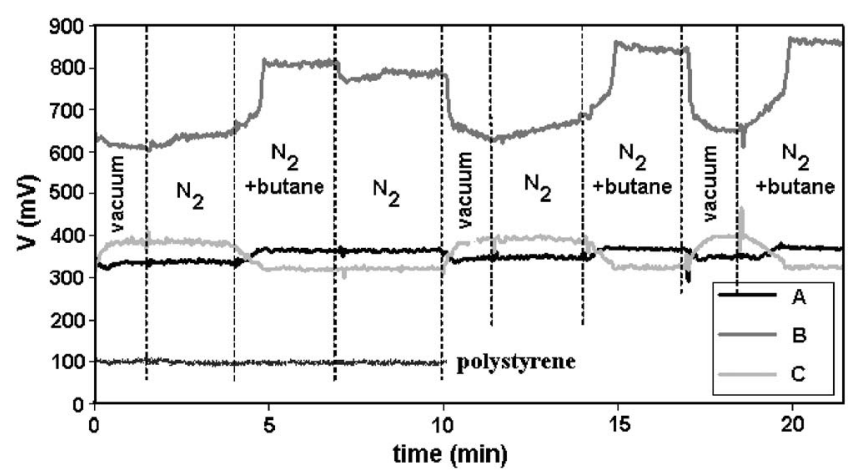

FIG. 4. Sensitivity of $\mathrm{ZnO}$ and polystyrene to $1000 \mathrm{ppm}$ of butane diluted in $\mathrm{N}_{2}$.

\section{B. Gas sensitivity tests}

All samples were exposed to different concentrations of butane diluted in nitrogen or air $(1000,500$, and $100 \mathrm{ppm})$ at room temperature. The protocol described in Sec. II was repeated twice to check the reproducibility of the response. A thin film of a nonsensitive material exposed to $1000 \mathrm{ppm}$ of butane was used as a control. The material was commercial polystyrene deposited by spray coating. This polymer is glassy at room temperature, and it is impossible for the gas molecules to diffuse into the film. There is no possible interaction between the phenyl groups of polystyrene and the hydrocarbon molecule. Typical optical responses are given in Figs. 4-9.

The optical response of $\mathrm{ZnO}$ was repeatable, as can be seen in Fig. 4. Vacuum stabilized the signal at $620 \mathrm{mV}$, while the mixture of butane stabilized it at $800 \mathrm{mV}$. By altering the concentration of the surrounding atmosphere, vacuum modified the balance of adsorbed species linked to the adsorbed oxygen ions at the surface of $\mathrm{ZnO}$, and therefore the refractive index of the film. This led to a signal variation of up to $27 \%$ for B-type samples. The balance remained unchanged when $\mathrm{N}_{2}$ was introduced, as vacuum was removed by a neutral gas (as observed in Fig. 4). The butane molecules interacted with the adsorbed oxygen ions on the surface leading to a variation in balance and thus in the refractive index. As butane was introduced just after the vacuum pumping, a signal variation was observed. The polystyrene control showed no optical response, as expected. This clearly proved that the signal variations were due to the presence of butane, and could not be attributed to pressure effects. Moreover, the

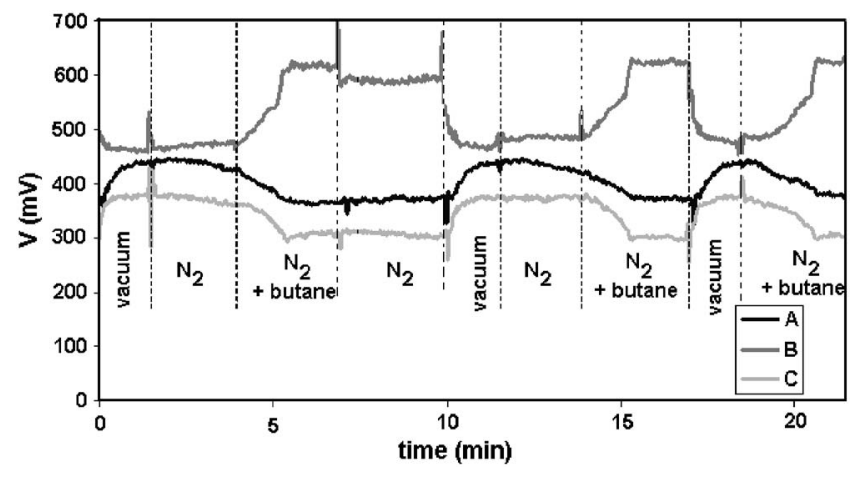

FIG. 5. Sensitivity of $\mathrm{ZnO}$ to $500 \mathrm{ppm}$ of butane diluted in $\mathrm{N}_{2}$.

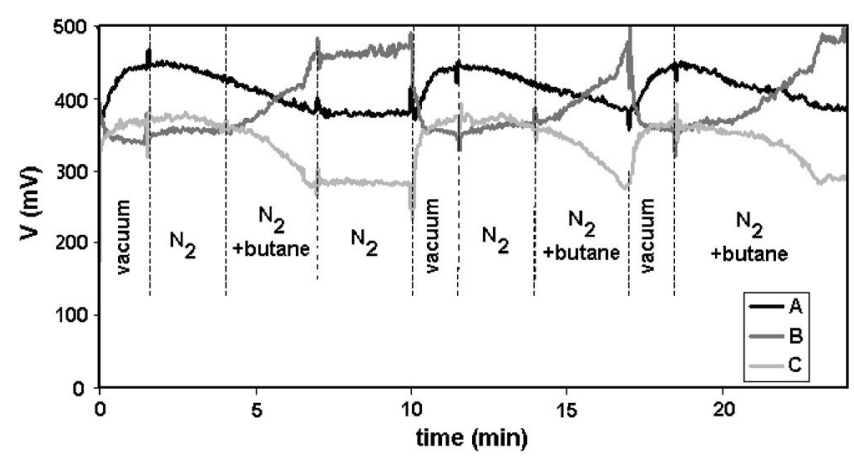

FIG. 6. Sensitivity of $\mathrm{ZnO}$ to $100 \mathrm{ppm}$ of butane diluted in $\mathrm{N}_{2}$.

signal always reached the same level regardless of the preceding phase of the protocol, which indicates high reproducibility and reversibility. The C-type samples showed a reverse optical response compared to those of the types A and B. Since the photodiode was always placed on the same profile slope, we concluded that the refractive index varied in the opposite direction. The reversibility of the response was due to vacuum pumping. The most common way to get a reversible sensor is to heat the film to desorb the gas molecules at the surface of the material.

All $\mathrm{ZnO}$ films showed similar responses when the concentration of butane decreased, as seen in Figs. 5 and 6. The shape of the signal variation remained the same, but the kinetics was slower at lower butane concentrations. The response time was about $1 \mathrm{~min}$ for $1000 \mathrm{ppm}, 2 \mathrm{~min}$ for $500 \mathrm{ppm}$, and $3 \mathrm{~min} 30$ for $100 \mathrm{ppm}$. The same signal levels were reached, probably due to saturation effects, for all but the C-type samples. We defined the response time as the time it takes to stabilize the signal level from carrier gas flow case to butane exposure case. As can be seen in Figs. 4-9, the detected signal was highly reproducible under stabilized conditions. There is no signal recovery if no external energy is brought to the film. The recovery time is therefore very long (probably several hours). Vacuum pumping ensures the desorption of molecules in our case. The concentration information could be estimated by derivation of the signal versus time.

For the sake of comparison, the samples were exposed to butane diluted in air. Similar responses were recorded for all samples. However, in order to provide clearly distinguishable curves in Figs. 7-9, we only represented the results for samples B and C. When air flowed in, oxygen was not ad-

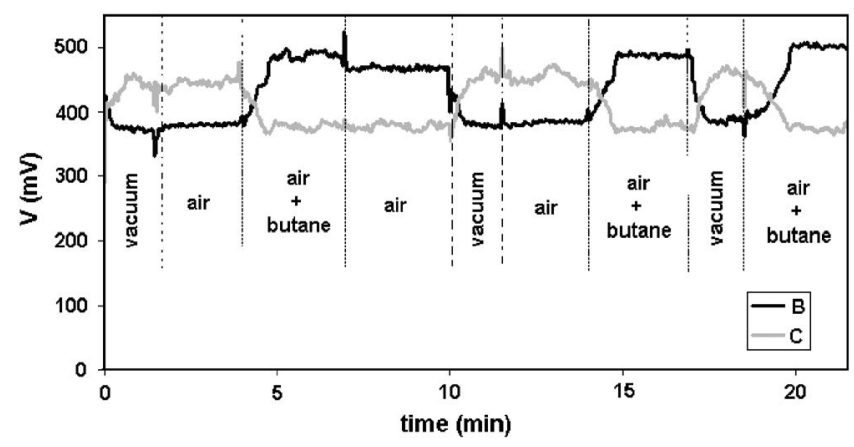

FIG. 7. Sensitivity of $\mathrm{ZnO}$ to $1000 \mathrm{ppm}$ of butane diluted in the air. 


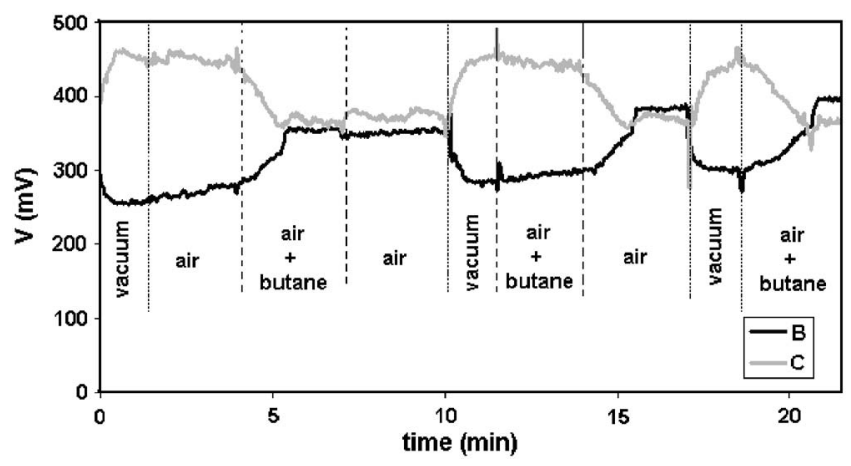

FIG. 8. Sensitivity of $\mathrm{ZnO}$ to $500 \mathrm{ppm}$ of butane diluted in the air.

sorbed at the surface. The signal variation only occurred when butane flowed into the cell. The signal variation amplitude and time response were the same as when nitrogen was used as carrier gas.

We evaluated the reproducibility of our method and procedure by exposing the tested structures to the same investigation cycle during two different periods of time: from 0 to $7 \mathrm{~min}$ and 10 to $17 \mathrm{~min}$, respectively (see Figs. 4-9).

We defined the reproducibility $R$ as

$$
R(t)=\frac{S(t+10 \min )-S(t)}{S(t)} 100, \quad t \in[0,7] \mathrm{min},
$$

where $S(t)$ is the signal recorded at moment $t$. As a typical example, we gave in Fig. 10 the calculated $R(t)$ values for sample A and two butane concentrations of 500 and 1000 ppm, respectively. We observed an excellent reproducibility of results, as illustrated by accuracy better than $\pm 5 \%$. Small variations were only visible during the short transition times between the different phases of the protocol.

We calculated (Table II) the variation in effective index of the different layers with Eq. (2). The refractive index of the prism $n_{p}$ was 2.5821 , and its angle $A_{p}=45^{\circ}$. Using the profile described in Fig. 11, $\Delta \theta_{s}$ was deduced from the linear shift $\Delta d$ of the line measured by the photodiode. $\Delta \theta_{s}$ is linked to $\Delta N$ by the following equation:

$$
\Delta \theta_{s}=\frac{\Delta d}{D}
$$

where $D$ is the distance between the prism and the photodiode $(D=0.75 \mathrm{~m}$ in our case).

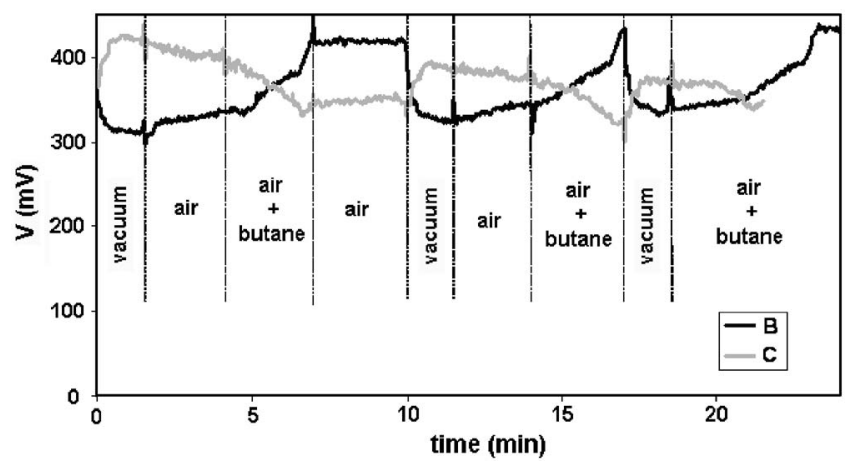

FIG. 9. Sensitivity of $\mathrm{ZnO}$ to $100 \mathrm{ppm}$ of butane diluted in the air.

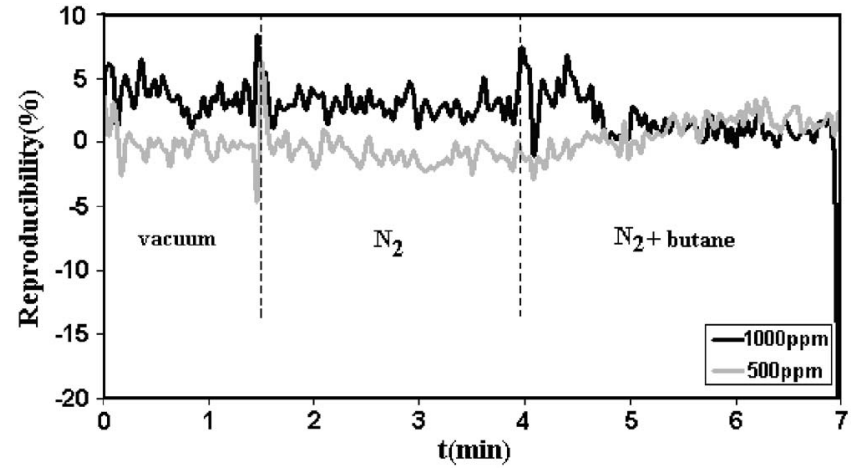

FIG. 10. Typical response reproducibility curve for sample A in the case of butane concentration in nitrogen of 500 and $1000 \mathrm{ppm}$.

For $\mathrm{A}$, the signal variation ranges between $V_{\min }$ $=304 \mathrm{mV}$ and $V_{\max }=384 \mathrm{mV}$, whereas for $\mathrm{B}$, it ranges between $V_{\min }=650 \mathrm{mV}$ and $V_{\max }=850 \mathrm{mV}$ (see Fig. 4). In spite of the difference of the dynamic of the signal, it corresponds for each sample to a linear shift of $0.1 \mathrm{~mm} . \Delta \theta_{s}=1.33$ $\times 10^{-3} \mathrm{rad}=0.0076^{\circ}$.

We noticed that signal B oscillations in Fig. 11 were caused by an interference of the reflected beam. Indeed, interference fringes arise due to numerous internal reflections in prism and other interfaces. This phenomenon is particularly obvious in the case of synchronism angles close to normal incidence (from $-10^{\circ}$ to $+10^{\circ}$ ).

Table II shows that the sensitivity of the films is linked to the angular shift of the line rather than signal levels. Effective index variations down to 0.005 could be detected for both A- and B-type samples, but the signal variations were more important for the B samples (see Fig. 4). The samples of type A therefore had the same sensitivity in terms of effective index variation. This proves that it is important for a layer with high-optical properties to provide a high-contrast line that can facilitate the observation of low variations in the refractive index.

As for the $\mathrm{C}$ samples, the effective index variation was the same, not being influenced by the carrier gas, probably because the alignment of the photodiode was the same during the two tests.

\section{CONCLUSIONS}

$\mathrm{ZnO}$ thin films deposited by PLD were optically characterized using the $m$-lines technique. The refractive index was in the 1.95 region. The films exhibited a slight anisotropy. They were exposed to different concentrations of butane diluted in nitrogen and air. A large optical response, i.e., a signal variation corresponding to a refractive index material

TABLE II. Variation in the effective index of the $\mathrm{ZnO}$ films.

\begin{tabular}{cccccc}
\hline \hline Sample type & Carrier gas & Mode & $\theta_{s}\left(^{\circ}\right)$ & $\Delta \theta_{s}\left(^{\circ}\right)$ & $\Delta N$ \\
\hline $\mathrm{A}$ & $\mathrm{N}_{2}$ & TM0 & 11.642 & 0.0076 & 0.005 \\
$\mathrm{~B}$ & $\mathrm{~N}_{2}$ & $\mathrm{TM} 0$ & 8.832 & 0.0076 & 0.005 \\
& air & & & 0.0267 & 0.018 \\
$\mathrm{C}$ & $\mathrm{N}_{2}$ & TM1 & -17.670 & 0.0154 & 0.012 \\
& & & & 0.0154 & 0.012
\end{tabular}




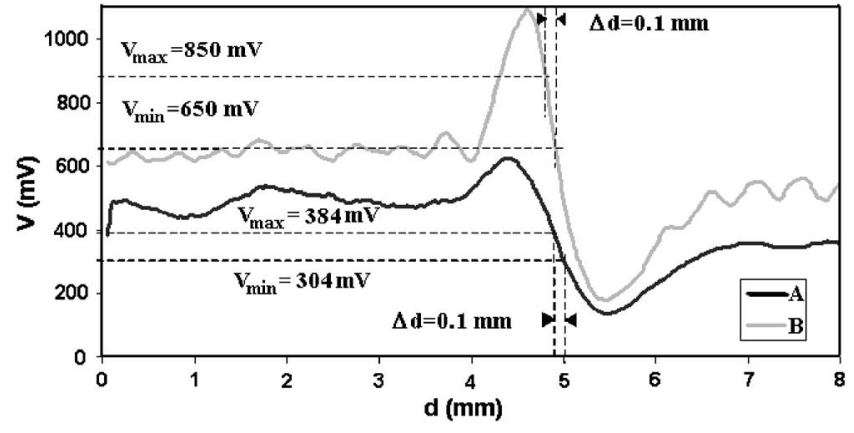

FIG. 11. $M$-line profiles of $\mathrm{ZnO}$ films.

change, was observed independently of the carrier gas for samples B and C. Effective index variations down to 0.005 were detected for samples A and B during butane exposure at room temperature. The obtained structures preserve gas sensitivity down to the lowest temperatures used in deposition, i.e., $20^{\circ} \mathrm{C}$ (type-C films).

We expect improvements in the case of integrated devices by elimination of the losses in light coupling mismatch of investigated films.

\section{ACKNOWLEDGMENT}

The authors acknowledge with thanks the financial support of the EU under the contract "Nanostructured photonic sensors,” NANOPHOS IST-2001-39112.

${ }^{1}$ S. S. Sarkisov, D. E. Diggs, G. Adamovsky, and M. J. Curley, Appl. Opt. LP40, 349 (2001).

${ }^{2}$ P. Liu, S.-H. Lee, H. M. Cheong, C. E. Tracy, J. R. Pitts, and R. D. Smith, J. Electrochem. Soc. 149, H76 (2002).
${ }^{3}$ Z. A. Ansari, R. N. Karekar, and R. C. Aiyer, Thin Solid Films 301, 82 (1997).

${ }^{4}$ K. Keis, C. Bauer, G. Boschloo, A. Hagfeldt, K. Westermark, H. Rensmo, and H. Siegbahn, J. Photochem. Photobiol., A 148, 57 (2002)

${ }^{5}$ S. F. Yu, C. Yuen, S. P. Lau, Y. G. Wang, H. W. Lee, and B. K. Tay, Appl. Phys. Lett. 83, 4288 (2003).

${ }^{6}$ J. Xu, Y. Shun, Q. Pan, and J. Qin, Sens. Actuators B 66, 161 (2000).

${ }^{7}$ X. L. Cheng, H. Zhao, L. H. Huo, S. Gao, and J. G. Zhao, Sens. Actuators B 102, 248 (2004).

${ }^{8}$ N. Koshizaki and T. Oyama, Sens. Actuators B 66, 119 (2000).

${ }^{9}$ S. K. Hong, Y. Chen, H. J. Ko, H. Wenisch, T. Hanada, and T. Yao, J. Electron. Mater. 84, 3912 (1998).

${ }^{10}$ T. M. Barnes, J. Leaf, C. Fry, and C. A. Wolden, J. Cryst. Growth 274, 412 (2005)

${ }^{11}$ D. J. Kang, J. S. Kim, S. W. Jeong, Y. Roh, S. H. Jeong, and J. H. Boo, Thin Solid Films 475, 160 (2005).

${ }^{12}$ S. J. Henley, M. N. R. Ashfold, and D. Cherns, Surf. Coat. Technol. 177-178, 271 (2004).

${ }^{13}$ X. W. Sun and H. S. Kwok, J. Appl. Phys. 86, 408 (1999).

${ }^{14}$ Y. Sun, G. M. Fuge, and M. N. R. Ashfold, Chem. Phys. Lett. 396, 21 (2004).

${ }^{15}$ V. Craciun, S. Amirhaghi, D. Craciun, J. Elders, J. G. E. Gardiniers, and I. W. Boyd, Appl. Surf. Sci. 86, 99 (1995).

${ }^{16}$ E. György, G. Socol, I. N. Mihailescu, J. Santiso, C. Ducu, and S. Ciuca, Proc. SPIE 5830-06, 50 (2005).

${ }^{17}$ Y. Ma, W. L. Wang, K. J. Liao, and C. Y. Kong, J. Wide Bandgap Mater. 10, 113 (2002).

${ }^{18}$ J. Q. Xu, Q. Y. Pan, Y. A. Shun, and Z. Z. Tian, Sens. Actuators B 66, 277 (2000).

${ }^{19}$ S. Agan, F. Ay, A. Kocabas, and A. Aydinli, Appl. Phys. A: Mater. Sci. Process. 80, 341 (2003).

${ }^{20}$ J. Shi, Z. Cao, J. Zhu, and Q. Shen, Appl. Phys. Lett. 84, 3253 (2004).

${ }^{21}$ Y. Luo, D. C. Hall, L. Kou, O. Blum, and H. Hou, Appl. Phys. Lett. 75, 15 (1999).

${ }^{22}$ F. Prieto, A. Llobera, D. Gimenez, C. Domengez, A. Calle, and M. Lechuga, J. Lightwave Technol. 18, 966 (2000).

${ }^{23}$ S. Monneret, P. Huguet-Chantôme, and F. Flory, J. Opt. A, Pure Appl. Opt. 2, 188 (2000).

${ }^{24}{ }_{\text {www.intlsensor.com }}$ 